\title{
About one approach to building low latency network file system
}

\author{
Dmitry Irtegov \\ Assistant prof. of Novosibirsk State \\ University, Russia \\ 630090 Pirogova 2 \\ Novosibirsk, Russia \\ +73833634339 \\ fat@nsu.ru \\ Sergey Oleynikov \\ Director of Development, Parallels \\ inc \\ 630007 Oktyabrskaya Magistral, 4, \\ Novosibirsk, Russia \\ +7 (383) 3637101 ext.76141 \\ soleynikov@parallels.com
}

\author{
Igor Knyazev \\ Master student of Novosibirsk State \\ University, Russia \\ 630090 Pirogova 2 \\ Novosibirsk, Russia \\ +73833634339 \\ knyazev.igor.nsk@gmail.com \\ Michael Rootman \\ Teaching assistant of Novosibirsk \\ State University, Russia \\ 630090 Pirogova 2 \\ Novosibirsk, Russia \\ +73833634339 \\ mrootman@mail.ru
}

\author{
Julia Mallaeva \\ Master student of Novosibirsk State \\ University, Russia \\ 630090 Pirogova 2 \\ Novosibirsk, Russia \\ +73833634339 \\ heimaolee@gmail.com \\ Dmitry Solovyev \\ Performance \& Security \\ Development Manager, Parallels inc \\ 630007 Oktyabrskaya Magistral, 4, \\ Novosibirsk, Russia \\ +7 (383) 3637101 ext.76112 \\ dsolovyev@parallels.com
}

\begin{abstract}
In this paper we describe an approach to building low latency shared network storage based on multi-layered unification and results of testing a prototype of this type of storage.
\end{abstract}

\section{Categories and Subject Descriptors}

D.4.3 [File Systems Management]: Access methods, Distributed file systems,

\section{General Terms}

Performance

\section{Keywords}

Network shared storage, network accessed storage, network file system, shared storage, latency, relaxed consistency, multilayered unification, shared web-hosting, load balancing.

\section{INTRODUCTION}

File system performance is described by large number of parameters [1]. Depending on type of access (proportion of read and write operations; pattern of random or sequential access; pattern of storage allocation, etc) throughput and other parameters of file system can vary $[2,3]$.

Permission to make digital or hard copies of part or all of this work for personal or classroom use is granted without fee provided that copies are not made or distributed for profit or commercial advantage and that copies bear this notice and the full citation on the first page. Copyrights for thirdparty components of this work must be honored. For all other uses, contact the Owner/Author.

Copyright is held by the owner/author(s).

CEE-SECR '14, Oct 23-25 2014, Moscow, Russian Federation ACM 978-1-4503-2889-0/14/10.

http://dx.doi.org/10.1145/2687233.2687248
For different applications different parameters are important. Most benchmarks concentrate on throughput, but for some applications latency is more important.

For example, work of server side of typical web application consists in opening large numbers of small files with average size of several kilobytes or tens of kilobytes. Most of these files by number and volume are server-side script libraries used by main page script. Latency when opening these files can have noticeable impact on performance of the application. This puts constraints on selection of a file system for web servers, especially if we try to select a network shared storage. Request for networked shared storage for web servers appears in the context of load balancing.

This allows to formulate a task to develop a specialized file system or file storage for web servers.

One possible approach to building such a filesystem is based on the assumption that typical web application opens most of the files only for reading. This allows building a virtual file storage consisting of two branches. One branch is available for readwrite and provides shared access and consistency and other is available only for reading and provides low latency and high throughput. Patterns of web application file access allow to relax consistency reqirements of this type of storage enough that this scheme would provide an average latency determined by readonly branch.

This article discusses feasibility and probable advantages of this type of file storage in comparison to alternatives available on the market. Also we describe working prototype of storage of this type and results of its performance testing.

Storage of this type can be attractive for other tasks that include opening of large numbers of small files, like compilation or automated software testing. 


\section{WEB SERVERS AND FILE SYSTEMS}

Web applications that were developed with consideration for load balancing, such as google.com or facebook.com, do not use file system for storage of application state [4]. In these applications, all state is stored in a central or distributed database. File system contains server-side code of application itself (usually scripts written on some dynamic language) and static web resources - pictures, .css files, JavaScript, etc.

Applications built with this approach use file system only for reading and they are easy scalable for load-balanced clusters. Update of code and static content happens in centralized manner, under control of cluster administrator, and can involve even gradual rebooting of cluster nodes when they switch to a storage with new version of content.

As we will see, this opens very wide possibilities for optimization of both throughput and latency of the file system, up to the point that makes this area not interesting for research.

Also, this makes load balancing relatively easy: because all application state is shared between all nodes, switching an application session to another node involves only redirection of HTTP requests.

With applications used on typical shared web hosting, situation is very different. Applications used by clients of shared hosting are often developed without scalability in mind. Because shared hosting is mostly used for low-traffic sites, non-scalability is often justified, because most of these sites will likely never need to scale. But shared hosting providers do need to scale, and behavior of hosted applications puts limits to this.

In this article we will call an owner of a web-site, placed on shared web hosting, a [hosting] client, and a person or application accessing a web-site - an user.

The main problem is that many web applications, including most popular ones, such as WordPress, Joomla! and Gallery, use file system for storing [parts of] their state. Also, hosting clients update code and static content of their applications without notifying a hosting operator and they use wide set of tools to do the update. So the hosting operator is forced to give them full read-write access to areas of the server filesystem where clients code and data are stored.

Building a load balancing system for this kind of applications is a complex task that requires a multi-view research $[5,6]$. One of the key problems is a transfer or sharing of consistent application state between the source and target nodes. Because part of state is placed on the filesystem, a good solution of this problem might also solve problems that will arise if client would start updating the application when it is being transferred.

Let us briefly discuss logically possible approaches for solving this problem.

\subsection{Copying or replicating application state}

In typical shared hosting, application state and static resources are placed on local hard disks of the server nodes. This allows best possible latency and throughput, but transferring application state to other node involves remote file copy of all application files.

There exist tools for optimized remote copying (replication), such as rsync [7], so it's theoretically possible to keep out of sync copies of applications on all nodes and replicate its current state only during the transfer. This will likely save time during the copy, but would create a background task of updating all copies so they won't get too far out of sync.

In any case, remote file copy is an expensive operation in terms of time, network traffic and node CPU load. Because load balancing usually occurs during high load on source node, this looks unattractive.

Another problem is that state of application during the copy is not consistent. Probably the only effective solution for this is to stop the application for duration of the copy.

Last but not least, every node in a cluster must have enough disk space to accommodate all applications in this cluster. In large clusters this can increase disk space requirements by order of magnitude in comparison with the other approaches.

\subsection{Placing the application on exclusive access network storage}

Modern network attached storage systems (NAS) provide latencies and [theoretical] throughput comparable to local hard disks, so in modern datacenters these systems rapidly displace directly attached storage.

Typical use of NAS involves exporting a raw disk space. For storage node this space might be actually a file, but server node sees it as a [virtual] block device, accessible by such protocols as iSCSI, AoE, etc. Server nodes place traditional file system such as ext3/ext4 on this block device and use it no different from local hard disk.

Traditional filesystems do not tolerate shared read-write access to a storage. Filesystem drivers have unprotected critical sections when working with on-disk structures. Race conditions in these sections will lead to corruption of a filesystem metadata.

To transfer exclusive NAS storage from one node to another we must unmount it on source node and mount on target one. This is several orders of magnitude faster and cheaper than copy, but still takes from seconds to minutes depending on the storage volume, caching state and other circumstances.

Linux umount [8] with default options will never successfully finish if there are open files on the volume. Forced umount will lead to file operations returning error codes, which, in turn, will disrupt operation of web server processes up to the crash. So we must disable not olny migrated application, but probably entire node for the duration of the transfer.

Approaches used for live migration of virtual machines and virtual private environments are not directly applicable here. When migrating a virtual machine, we transfer all its state together with in-memory parts of application state, web server processes state, OS and filesystem driver in-memory state, etc., so we do not need to serialize all this on disk.

Here we must migrate part of web server processes in-memory state, related to serving migrated application, leave all other parts of server state on source node undamaged, and integrate migrated state into the state of target server, again leaving all other parts of that state undamaged. In this context, we consider in-memory state of FastCGI daemons and similar services to be a 
part of web server state. Modern web servers are not adapted to this kind of partial state transfer and their adaptability is, at least, not proven.

So, implementing a migration of web applications based on switching exclusive NAS storage is much better than copy or replication, both in terms of migration cost and storage space, but it is still not a live migration. Implementing live migration in this style is very challenging and probably unsolvable task.

\subsection{Using shared network storage}

From migration perspective, this approach seems to be ideal. All application state is placed in a single storage available to all nodes, and this storage always contains the current copy of the state. Migration process is as simple as in scalable applications and involves only redirection of HTTP requests

Theoretically, there could arise race conditions in applications not designed for execution on several nodes in parallel. But all modern web servers are multiprocessed and most are also multithreaded, and they usually make no provisions that requests to a single application are served in single process. So all web applications already must be designed and tested to be racecondition proof. Possibly existing race conditions would also manifest themselves in single-node environment.

So, live migration, when application continues to serve old HTTP requests on source node and new requests are redirected to target node, is possible.

However, all popular types of shared network storage have performance properties that make them suboptimal choice for serving web applications.

\section{TYPES OF SHARED STORAGE AND THEIR PERFORMANCE PROPERTIES}

Available types of shared network storage can be divided to two main categories:

1. Network file systems

2. Cluster file systems

Network file systems use a special network protocol. This protocol is some type of remote procedure call interface, usually more or less directly mappable to OS file access API. This RPC contains calls to open file, read and write it and do other FSrelated operations [9]. In Unix-like OS most popular protocol of this type is NFS [10].

Term "cluster file system" has no generally accepted definition. In this article we will use this term to describe filesystems similar to OCFS2 [11] and GFS2, because OCFS uses words "cluster file system" as part of its name.

Cluster filesystems use raw network storage, such as iSCSI, but use it in nonexclusive (shared) manner. Cluster FS protect modifications of on-disk data and metadata on shared storage by special locking protocol. In OCFS2, this protocol and corresponding service process are called DLM (distributed lock manager). The DLM protocol itself is based on network exchanges between all nodes of the cluster.

Because of the locking protocol, cluster FS can tolerate parallel modification of storage by different nodes, so they often can serve as replacement for network file systems. In other respects, they are not much different from traditional file systems.

In table 1 are presented data of performance of web application (WackoWiki) with different file storages used for storing application files. All tests were done on same hardware and same database server.

Table 1. Access times of web server page on different storage types (excerpts from Apache Jmeter aggregate reports, 50 samples, all times in milliseconds)

\begin{tabular}{|c|c|c|c|c|}
\hline Storage & average & $90 \%$ line & $\min$ & $\max$ \\
\hline HDD/ext4 & 10 & 9 & 6 & 87 \\
\hline iSCSI/ext4 & 9 & 10 & 5 & 138 \\
\hline $\begin{array}{c}\text { iSCSI/OCFS2 } \\
\text { single node }\end{array}$ & $\begin{array}{c}9-10 \\
\text { estimation }\end{array}$ & & & \\
\hline SMBFS & 179 & 193 & 163 & 195 \\
\hline NFS & 19 & 19 & 14 & 150 \\
\hline NFS nocto & 8 & 10 & 8 & 14 \\
\hline
\end{tabular}

WackoWiki stores main bulk of its data in MySQL database. In all tests, the same database located on different node was used. However, WackoWiki also uses a filesystem to store cache of documents converted from internal wiki format to HTML. This cache is located in subdirectory of the directory containing application scripts, and it can grow pretty large. This cache is the main reason this application was selected for tests.

WackoWiki is an example of badly behaving application, and it creates write requests on all attempts to open a wiki page. This makes it good target for creating a mixed read-write load.

Rows HDD and iSCSI/ext4 show times for locally attached HDD and iSCSI exclusive storage. iSCSI target was a CentOS 5 server with SATA disks and stock tgtd target server, iSCSI volume was a previously allocated file on JFS file system. In both cases ext4 filesystem was used.

Later in this article we will use exclusive NAS speed as a baseline that designates the best achievable performance. Comparing network storage to locally attached HDD is obviously unfair, and performance difference between NAS and HDD is negligible. Unfortunately, Apache Jmeter aggregate report does not output a standard deviation of the average value, but given min, max and $90 \%$ line values could help to estimate a deviation. Unfortunately, we do not have OCFS2 results on the same hardware, so only extrapolation derived from other measurements is included.

SMBFS produces results that are included only to show how bad it can go in practice.

Really interesting data are NFS row. It can be seen that NFS with default mount options is measurably worse than the other kinds of storage. The nature of this difference is easy to discover if we measure time of executing open system call (see table 2).

Table 2 Performance parameters of different network storage types under synthetic load

\begin{tabular}{|l|l|l|}
\hline Parameter & NFS & iSCSI/ext4 \\
\hline Open execution time & $30-40$ мс & $30-40$ мкс \\
\hline $\begin{array}{l}\text { Open execution time on saturated } \\
\text { network }\end{array}$ & $1-1.5 \mathrm{c}$ & 400 мкс \\
\hline
\end{tabular}




\begin{tabular}{|l|l|l|}
\hline $\begin{array}{l}\text { Throughput reading files of size } \\
2 \mathrm{~kb} \text { over saturated network }\end{array}$ & 722 б/сек & 17 Кб/сек \\
\hline
\end{tabular}

When WackoWiki opens the page, it executes a PHP script that, in order, uses PHP include function to open and read 32 other files with aggregate size around $200 \mathrm{~kb}$. Time to read these files from local HDD or from NAS over gigabit network is negligible compared to script execution and database access time, so we do not see difference between directly attached and networked storage on our test.

However, on NFS time of opening these files is three orders of magnitude bigger than on other storages, and this time is dominating over the other sources of latency.

To prove that source of difference is open latency and not throughput, we included data on NFS mounted with nonstandard (and in the past undocumented) option nocto, present in Linux implementation. This option turns off a standard NFS method of keeping consistency of file metadata, so called close-to-open semantics [12], and switches to non-standard heuristic method of synchronization.

Option nocto brings open latency and web site performance to values comparable to exclusive NAS and local HDD.

Option nocto is poorly documented, and, as can be noticed from messages in kernel mailing lists, poorly supported and maintained. For example, in CentOS 6 this option works on NFS3 but not on NFS4, but this is never mentioned in documentation.

Most NFS guides don't even mention this option, and those documents that do either suggest to use it on read-only volumes [13] or suggest complex schemes for separating files with different access patterns on different NFS shares, and creating a complex set or symlinks to maintain directory structure [14].

Behavior of OCFS2 also needs more discussion. On read-only access or in single-node configuration, OCFS2 and similar cluster FS produce latencies and throughput comparable to exclusive NAS with traditional FS (see Figure 1). So these filesystems are actually used in web clusters.

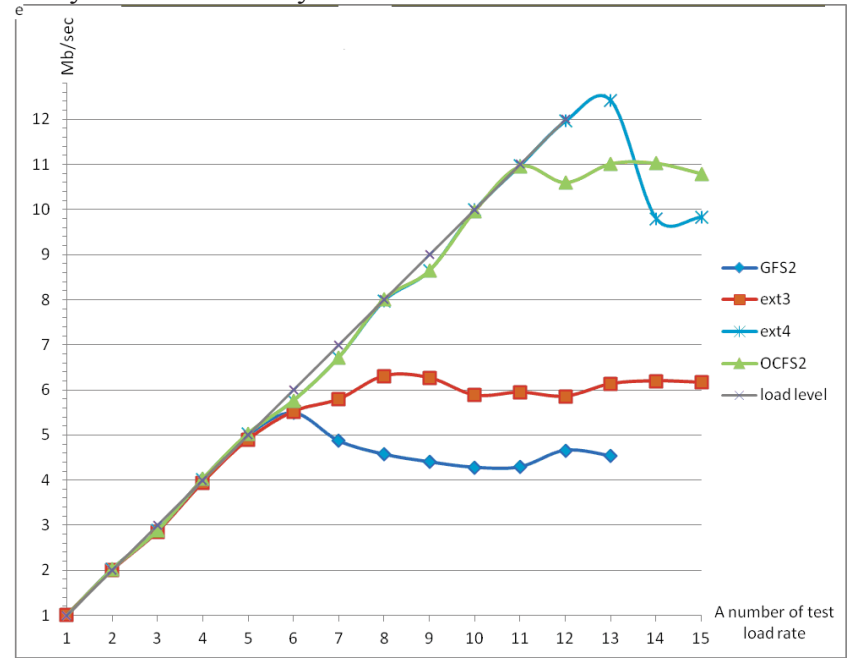

Figure 1 Throughput of single-node GFS2, OCFS2 and traditional file systems (synthetic benchmark).

However, when we use several nodes and mix of read-only and read-write accesses, we see measurable degradation.

On Figure 2 are presented the results of GFS2 and OCFS2 performance measurement in multinode configuration.

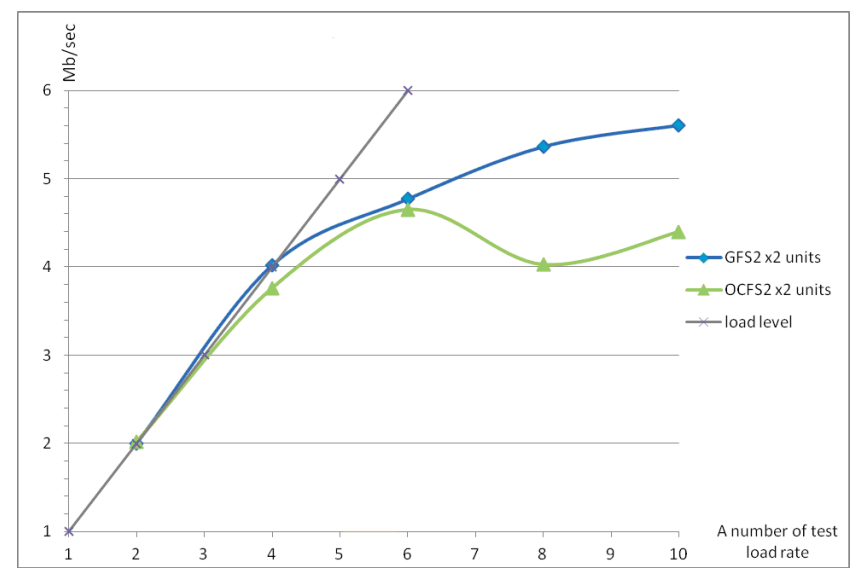

Figure 2 Throughput of cluster file systems in two-node setup.

Load was generated by xdd utility with following load parameters:

$50 \%$ of files have size $2 \mathrm{~kb}$ and open for read

$45 \%$ of files have size $30 \mathrm{~kb}$ and open for read

$5 \%$ of files have size $8 \mathrm{Mb}$ and open for read

$0.5 \%$ of files have size $30 \mathrm{~kb}$ and open for write

"Number of test load rate" designates a number of threads doing the load.

Theoretical explanation of this degradation is rather obvious. Indeed, cluster FS use separate locking protocols to ensure consistency of metadata. Distributed locking requires getting a confirmation from all nodes that they have received a locking request. Latency of this confirmation is the source of latency for other types of filesystem operations.

Latency of distributed locking is a well-known problem in many kinds of distributed systems [15] and there is no known universal solution for this.

It is important to note that while locking latency occurs only when write load is present, it also influences read operations. By nature of locking, when a node requests a lock on a on-disk structure, it forbids not only modifying but also reading this structure.

So, while OCFS2 is better than NFS with default mount options, there is still a possibility of better solution.

\section{STORAGE WITH MULTI-LAYERED UNIFICATION}

Data and reasons presented above lead us to the idea that possible solution for optimal storage structure could be found by relaxing consistency criteria for shared storage. Data on NFS nocto show what can be achieved by this approach.

However, we must find a relaxation of consistency rules such that it would not damage web applications and at the same time would allow us to achieve a performance gain.

The research of behavior of popular web applications used on shared hosting, such as WordPress, Gallery, Joomla!, Drupal, etc shows that even when these applications place their data on filesystem, most file accesses occur in read-only mode. Synthetic 
load used for obtaining Figure 1 data reflects Parallels estimate of actual shared hosting file access patterns.

Also, files with write access fall in two distinct categories. Most of the writable files are written once and never modified again. For example, some of mentioned applications allow users to upload images and media files, and place them directly on filesystem.

The second category by frequency are files that are always opened for writing. Examples of these files are PHP session files and local databases such as SQLite or BerkeleyDB.

This brings us to the idea of dual storage system consisting of two branches. Both branches are shared storage accessible to all cluster nodes.

One branch is read only. It stores static content and parts of application state that were modified long ago and will likely never be modified again. The other branch is read-write, provides write consistency and stores files that are or can be opened for writing.

Because most files are opened on RO branch, average latency, average throughput and web application performance would be defined by parameters of $\mathrm{RO}$ branch. We have seen that there are many options for low latency RO shared storage.

However, this idea creates two other problems:

1. Typical web applications do not divide and do not specially mark files with different patterns of access. There are some heuristic methods of separating them but heuristics, by definition, are prone to errors.

2. Static content is modified from time to time. It must be migrated to RW branch and this eventually will lead to all files moving to RW branch.

Solution of the first problem is well known and called multilayered unification. Multi-layered unification is the recursive joining of two or more filesystems, called branches (note that we already introduced this term above).

Joining is implemented in such a way that if there are directories with same hierarchical names on two branches, content of resulting directory will be the union (in set theory sense) of original directories. If files with same hierarchical name exist in two branches, selection of the file will be defined by a branch priority.

For Linux, there exist at least two virtual filesystem drivers implementing multi-layered unification, UnionFS [16] and AUFS [17].

Most known application of these filesystems are bootable $\mathrm{CD}$, so called "Live CD". Such a CD contains a read-only image of filesystem. When the CD boots, it creates a RAM disk and joins it with content of the CD. Modified files are placed on RAM disk on first modification, and it creates the illusion of normal Unix system with read-write filesystem.

Actually, both UnionFS and AUFS can use persistent storage for RW branch, join multiple RO branches and do other interesting stuff. But for our task these filesystems are not suitable for two reasons.

First, both AUFS and UnionFS check existence of a file on RW branch before accessing all other branches. So, open latency of resulting filesystem will be defined by the RW branch, and this is exactly what we are trying to avoid.

Second, on both named filesystems, the RW branch starts in empty state. When you first open file from RO branch for writing, it is being copied on RW. This also has a big impact on latency and is not acceptable for us.

In our proposed multi-layered unification system that we develop under name PLESK-FS, a branch where a file is located is determined by special data structure that we call translation table (TT).

Translation Table is a hash table of inode numbers of all files that were opened for writing since some moment in the past (selection of this moment will be discussed below). It might also contain files that have high estimate of probability of being opened for writing.

Files listed in TT are always opened on RW branch. Files that are opened for writing for the first time are included in TT and also opened on RW branch. Files that are not listed in TT and are being opened for read are opened on RO branch. Deleted files are marked by special flag in TT that makes them inaccessible, but they continue to exist on RO branch. So, hash table is checked on every file open.

A copy of TT is stored in memory of all cluster nodes accessing PLESK-FS volume. In current implementation we store it in kernel memory. This ensures low latency.

TT is modified when a node executes one of the following operations: renaming or deleting file, changing its attributes, and, as it was said above, when file is first opened for writing. The changes in TT are pushed by node that introduced them to all other nodes.

The main idea of this scheme is that we do not wait for other nodes to receive our change and to confirm that. In this fashion, we avoid a strong source of latency. But this behavior is actually a relaxed consistency, or, more specifically, eventual consistency. We guarantee that all TT changes will be propagated to all nodes (details will be discussed in following section), but we cannot guarantee when that will happen. And we not wait for confirmation of this.

So there is a possibility that two nodes will do different operations on a same file when TT change is pending.

Let us consider possible scenarios of conflicts that this relaxation could create.

First, consider a scenario when two nodes open the same file for writing for the first time. It will be redirected to the same inode on RW branch, and the same (duplicating) record would be created in TT. Because opens will be redirected to the same object, this is not a problem. We just must make a provision that duplicate records in TT are joined.

This also includes two subscenarios, when two nodes create a file with the same name and when nodes are attempting to create temporary files with unique names using O EXCL flag of open system call. In all cases, these operations will be redirected to RW branch and will be handled by this branch according to standard POSIX semantics.

Second, consider a scenario when one node opens a file and second node deletes it. The first node will keep access to the file, but the file will be made unaccessible for further opens. 
This actually mimics a standard "lazy delete" behavior of Unixlike systems that allow deleting opened files with exactly the same resulting behavior.

Third scenario is when one node is opening a file for reading on RO brahch and second node is opening this file for writing for a first time. The file will be moved to RW branch, but the first node will not notice this and will continue to work with obsolete content.

Third scenario is the most dangerous and the only actually deviating from POSIX semantics. But we could not find examples of web applications that could be harmed by this.

First, in Unix, many file updates are made in "delete old file and create new with same name" style. This is what most Linux package managers and file archivers do. Updating a file in this style in combination with Unix "lazy delete" behavior would lead to the same results: processes that were holding an updated file will not see new content.

Second, web applications are asynchronous by their nature. In occasions when they do need synchronization, they use a database, and database files always are opened for read-write.

Now let us discuss how we solve a problem of hoarding of all content by RW branch and a problem of copying files from RO branch to RW.

In PLESK-FS initial state, RW branch is a master copy and it contains all files in the volume. RO branch is a snapshot of RW made at some moment by means of Linux LVM [18].

Periodically, for example, every midnight, we create a new snapshot of RW and make it a new RO branch. After this we rebuild a translation table. At this moment, we can apply heuristics, rules and statistical data that allow to conclude that it's better to place some files on RW from very beginning.

PLESK-FS file server, holding both branches, announces to cluster nodes that a new RO branch is available, and transfers a new translation table. Nodes mount a new RO branch and redirect newly opened RO files to it. However, the files that were opened during the update are continued to be served from old RO.

Every node monitors usage of the old RO branch. When all files on it are closed, node unmounts the branch and notifies the file server. When the server gets notifications from all nodes, it destroys the old branch.

It is easy to see that this scheme accommodates both applications storing user data in files and updates of the applications.

The application owner can notice performance degradation after update, but it should disappear after creation of new RO, so it can be listed as documented limitation of the technology.

The problem for this scheme are files that are held open for long time. "Long" in this context means for time comparable with lifetime of RO branch. Usually web applications do not have such files. Application script executes for short time when serving an HTTP request and closes all files when finishing.

However, let's consider a PHP script requesting a call of a function from application-supplied shared library. The function would complete in short time, but shared library image will be permanently attached to a process doing PHP execution. Also, sometimes clients of shared hosting are allowed to launch their own background or daemon processes. These processes can execute for long time and delay release of old RO branch.

Best solution would be creating a rule that would redirect all files used in such a manner to RW bransh. Also it must be noted that existence of such processes can be a problem for load balancing itself. For example, if application uses intra-node IPC to talk to application-supplied daemon, this application cannot participate in load balancing.

May be hosting operators would need to create dedicated servers and tariff plans for clients who require such processes, and explicitly forbid or limit these kinds of behavior for clients served by load balanced cluster.

\section{DESCRIPTION OF PLESK-FS PROTOTYPE AND PERFORMANCE DATA}

Authors implemented a prototype of described virtual file system PLESK-FS. Prototype consists of Linux kernel module (VFS driver), mount and umount utilities and components running on a file server. Because our goal was to demonstrate achievability of low latency, using FUSE driver was not an option even on early prototyping stage.

VFS module is written for kernel version 2.6.32 (version used in RHEL 6 and CentOS 6). All testing of kernel module and userland components was done on CentOS 6 x86_64. All code is written in $\mathrm{C}$ and we used good practices to avoid nonportable constructions, so there should be no problem porting it to other architectures.

VFS driver does not depend on nature of RW and RO branches. But logic of RO branch creation depends on the assumptions that RW content is placed on LVM logical volume and that snapshot of the volume can be somehow mounted by web server nodes in RO mode.

Most obvious solutions that fit to these assumptions are

1. RW branch is a traditional Linux filesystem shared via NFS or other network file system protocol. RO snapshot is exported via iSCSI or other NAS protocol.

2. RW branch is a cluster file system exported as shared NAS, and all web server nodes are members of the filesystem cluster. RO snapshot is exported via same NAS protocol, but mounted by web server nodes in single-node and/or readonly mode with distribute locking disabled.

In prototype we concentrated on first approach. File server is a dedicated node with ext4 volume exported by NFS. Snapshots of this volume are exported by iSCSI. Snapshot is done without umounting the original volume, but our experience shows that RO mounting of partially inconsistent ext 4 volume does not bring any noticeable degradation of performance.

This configuration was selected mainly because it was easy to setup. Also, because NFS with default mount options predictably has high open latency, it simplified the testing: you can guess what branch was used for any specific file just by measuring open time.

For small clusters it might be assumed that second approach with RW branch on cluster FS would provide better performance, at 
least for RW branch. But scalability of modern cluster FS on read-write access in big clusters with dozens or hundreds of web server nodes needs additional research, and our final goal is building a big cluster.

Most important component of PLESK-FS is the translation table. Essentially a PLESK-FS volume is a combination of RW branch, RO branch and a TT content, and correct operation of PLESK-FS is possible only if TT corresponds to state of branches.

A copy of TT is stored in kernel memory of every web server node. Changes in TT are pushed by two different network protocols, one called fast delivery protocol, second - guaranteed delivery.

Fast delivery protocol runs over UDP and consists in multicasting every change in the TT to all nodes.

Guaranteed delivery consists in communication with a central server over TCP connection. This architecture was selected because a hub and spoke topology contains much less links that fully connected network of links, so every web server node has to support less TCP sockets. There also are other reasons for central location of TT server that will be outlined below.

Every TT change is sent two times, via UDP and via TCP. This provides low average latency and guaranteed delivery. Note that this creates another reason to implement logic of removing duplicates in TT. Because TT is a hash table, this logic is pretty simple.

On web server nodes all communication is handled by kernel code linked together with VFS module. TT server is an userland daemon running on the file server node.

Besides pushing of changes in TT to all nodes, TT server fulfills other important functions:

1. It keeps the master copy of TT that is communicated to nodes joining to the cluster, and nodes reconnecting after losing a TT connection or a reboot.

2. It keeps the master copy of TT persistent during reboots of file server.

3. It informs cluster nodes about creation of the new RO branch, builds the new master TT during this creation and monitors usage of the old RO for removal.

4. It informs nodes joining the cluster about locations of $\mathrm{RO}$ and RW branches and about protocols for accessing them. In current prototype this functionality is limited and part of this information must be supplied as explicit parameters of mount command.

5. It allows nodes of the cluster not to know and not to care about total number of nodes, their IP addresses and other information. This contrasts to OCFS2 approach where IP addresses of all cluster nodes must be listed in configuration files of all nodes. This seems to be good for big clusters where reboots, adding, removing and replacing nodes could happen often.

Because creating of a new $\mathrm{RO}$ branch requires close interaction with the LVM of file server, the TT server must be placed on same node as a file server. This does not create a single point of failure, because NFS server and iSCSI target are already an unremovable single poitns of failure.

In current state of prototype, logic of migrating to a new RO is not fully implemented. Switching to a new RO branch is possible only when all RO files are closed and is done manually.

\subsection{Measurements}

Measurements on synthetic loads show that latency of opening files and throughput both on large and small files and their mix is no different from behavior of raw file systems where these files are placed. So we found no indication that our VFS layer is introducing additional sources of measurable latency.

We consider that a decisive experiment proving viability of PLESK-FS concept would be measuring a performance of a real web application deployed on PLESK-FS volume. This would prove that we both achieved correct implementation of core POSIX file API and set goals for performance.

Test stand was setup as a collection of identical Pentium DualCore E5200 2.50GHz computers, 2Gb RAM, 82801HR/HO/HH SATA, Seagate ST3160815AS HDD running CentOS 6.5 x8664. All computers were connected to single gigabit Ethernet switch.

One node was designated as a file server, and server components of PLESK-FS were deployed on it.

Another node was set up as web server by installing Apache2, PHP and other related packages from CentOS 6.5 repository. MySQL server for the web application was located on a separate computer. Because prototype in its current state does not support sendfile system call, we had to add parameter EnableSendfile off to Apache configuration.

WackoWiki 4.2 application was deployed to different types of storage on web server node and measured by Apache Jmeter. Reasons for selection of the applications were outlined in discussing data in Table 1.

In Table 3 are presented excerpts for Apache Jmeter aggregate report.

Table 3 Excerpts from Apache Jmeter aggregate report, 50 samples, all times in milliseconds

\begin{tabular}{|l|l|l|l|l|}
\hline Storage & average & $\mathbf{9 0 \%}$ _line & min & Max \\
\hline HDD & 23 & 26 & 22 & 28 \\
\hline iSCSI/ext4 rw & 23 & 24 & 22 & 30 \\
\hline NFS & 39 & 41 & 36 & 44 \\
\hline $\begin{array}{l}\text { PLESK-FS } \\
\text { RW }\end{array}$ & 39 & 40 & 37 & 44 \\
\hline $\begin{array}{l}\text { PLESK-FS } \\
\text { RO }\end{array}$ & 24 & 25 & 23 & 31 \\
\hline
\end{tabular}

Because testing was done on hardware different from one used to obtain data in Table 1, absolute values are different. It must be noted that this is not effect of disabling sendfile, because main performance impactor is time of loading and executing PHP scripts which is done via POSIX system calls.

It can be seen that difference between ext4fs and NFS is still very noticeable. Also it can be seen that HDD and exclusive 
iSCSI performance can be equally considered a baseline of best possible performance.

Row marked PLESK-FS RW is performance of the application right after the deployment, where all its files were placed on RW branch. This is worst possible scenario and it can be seen that performance is no different from raw NFS, which is known limitation of the approach.

Row marked PLESK-FS RO was obtained after generation of new RO image. This procedure both tested the functionality of RO switch and showed that performance increased up to the baseline.

It must be also noted that WackoWiki opens cache for read-write on each page access and corresponding files were correctly mapped back to RW branch which was checked by content of TT and by running similar tests with debug output enabled. But because only one cache file was used when generating the tested web page, opening latency of this file is not noticeable in aggregate results. This confirms original assumption that small number of high-latency files in the load will not impact the average performance.

\section{DISCUSSION AND CONCLUSIONS}

Measurements and tests show that virtual filesystem with multilayered unification and translation table can provide latency and throughput comparable with parameters of the branch where the corresponding file is located, and does it both on synthetic and realistic loads. Additional latency and decrease in throughput introduced by unification layer is deep inside of error margin.

To finally prove viability of the concept we should engage in more complex tests, including building large load-balanced cluster and deploying complex mix of web applications on it. This is topic of our further work, but the results obtained on this stage look very promising.

One possible direction of further work would also be modifying NFS caching scheme and cto/nocto semantics. If we introduce something similar to PLESK-FS translation table into NFS, we can open read-write files in cto mode and read-only files in nocto mode. Under conditions when majority of files are read-only, this would bring performance of NFS to levels comparable with exclusive NAS and better than cluster file systems. All this would be achieved without additional complexity of RO branch, RO branch switching and VFS unification layer. So this approach would yield result much more attractive for practical use.

However, this requires extensive modification if not reengineering of NFS kernel module. Chances that these modifications will be soon admitted into main Linux kernel source are very slim. Forking NFS kernel modules and offering the result for use in heavy-duty production environment is very risky from many perspectives. So we decided to postpone this direction of work until feasibility of our approach will be better proved.

\section{ACKNOWLEDGMENTS}

The work was implemented with financial support of Ministry of Education and Science of Russian Federation (contract № 02.G25.31.0054).

\section{REFERENCES}

[1] Лукьянов Н.М. Анализ факторов, влияющчих на качественные и количественные показатели функционирования систем распределенного хранения данных//Научно-технический вестник СПбГУ ИТМО. 2008. -№ 56. -9 c

[2] Нижник Е. И. Математическая модель нагрузки файловой системы NTFS при активном поиске дисковых данных//Моделирование процессов обработки информации. М.: Моск. физ.-техн. ин-т., 2007. С. 276285.

[3] Нижник Е. И., Тормасов А. Г. Очеека производительности файловой системы NTFS как задача множественной регрессии//Объединенный научный журнал, 2007. № 11 (199). С. 57-62.

[4] C. Henderson 2006. Building Scalable Web Sites: Building, Scaling, and Optimizing the Next Generation of Web Applications, O'Reilly Media.

[5] Сметанин А.Г., Тормасов А.Г. Математическая модель эффективных алгоритмов поиска и размещения данных в децентрализованной распределенной файловой системе, Научно-технические ведомости СанктПетербургского государственного политехнического университета. Информатика. Телекоммуникации. Управление. 2012. Т. 6. № 162. С. 16-22.

[6] Петров В.А., Тормасов А.Г., Миркин А.Л. Длительность миграџии виртуальных серверов в распределенной системе, Вестник Новосибирского государственного университета. Серия: Информационные технологии. 2009. Т. 7. № 1. С. 26-36.

[7] W. Davison, rsync web pages. Retrieved July, 2014 from http://rsync.samba.org.

[8] umount(8), Linux system manual. Retrieved July, 2014 from http://man7.org/linux/man-pages/man8/umount.8.html.

[9] Иртегов Д.В., 2008, Введение в операционные системы, 2-е изд, БХВ-Петербург,СПб.

[10] S. Shepler et al, Network File System (NFS) version 4 Protocol, RFC3530. Retrieved July, 2014 from http://www.ietf.org/rfc/rfc3530.txt.

[11] Project: OCFS2, Retrieved July, 2014 from https://oss.oracle.com/projects/ocfs2/.

[12] NFS(5), Linux system manual. Retrieved July, 2014 from http://man7.org/linux/man-pages/man5/nfs.5.html.

[13] NFS Options for Performance. Retrieved July, 2014 from http://www.dbaexpert.com/blog/nfs-options-forperformance/.

[14] Server performance tuning for Linux and Unix. Retrieved July, 2014 from

https://www.orcaware.com/svn/wiki/Server_performance_tu ning_for_Linux_and_Unix.

[15] A. Tannenbaum, M. Van Steen, 2006. Distributed Systems: Principles and Paradigms (2nd Edition), Prentice-Hall, Inc. Upper Saddle River, NJ, USA. 
[16] Unionfs: A Stackable Unification File System. Retreived July, 2014 from http://www.filesystems.org/projectunionfs.html.

[17] Another UnionFS. Retrieved July, 2014 from http://aufs. sourceforge.net/.
[18] lvm(8) Linux System Manual. Retrieved July, 2014 from http://man7.org/linux/man-pages/man8/lvm.8.html. 